\title{
The Distance Energy of Clique Trees*
}

\author{
Ya-Lei Jin ${ }^{1}$, Rui Gu${ }^{1}$, Xiao-Dong Zhang ${ }^{2}$ \\ ${ }^{1}$ Department of Mathematics, Shanghai Normal University, \\ 100 Guilin road, Shanghai, 200234, P.R. China \\ ${ }^{2}$ School of Mathematical Sciences, MOE-LSC, SHL-MAC, \\ Shanghai Jiao Tong University, \\ 800 Dongchuan road, Shanghai, 200240, P.R. China. \\ Email: yaleijin@shnu.edu.cn, gurui259011@163.com, xiaodong@sjtu.edu.cn
}

February 23, 2021

\begin{abstract}
The distance energy of a simple connected graph $G$ is defined as the sum of absolute values of its distance eigenvalues. In this paper, we mainly give a positive answer to a conjecture of distance energy of clique trees proposed by Lin, Liu and Lu [H. Q. Lin, R. F. Liu, X. W. Lu, The inertia and energy of the distance matrix of a connected graph, Linear Algebra Appl., 467 (2015), 29-39.].
\end{abstract}

Key words: Distance energy, Clique tree, Equitable partition, Spectral radius.

AMS Classifications: 05C50, 05C35.

\section{Introduction}

Graham and Pollak [12] in 1971 discovered an interesting and elegant result on the distance matrix of a tree that the determinant of distance matrix of any $n$-vertex tree is $(-1)^{n-1}(n-1) 2^{n-2}$, which is independent with the structure of trees. Furthermore, Graham and Lovász in 1978 [11] derived the coefficients of the characteristic polynomial of the distance matrix of a graphs in term of as certain tied linear combinations of the numbers of various subgraphs of a graph. Recently, Cheng and Lin [8] presented a class of graph whose distance determinant are independent of their structures. These results motivated that properties of distance matrix of a graph have been investigated. There are some excellent surveys (see [1, 3, 21]) on this topic.

Let $G$ be a connected graph of order $n$ with vertex set $V(G)$ and edge set $E(G)$. Thus the distance matrix of $G$ is defined as $D(G)=\left(d_{u v}\right)_{n \times n}$, where $d_{u v}$ is the distance between vertices $u$

\footnotetext{
${ }^{*}$ This work is Supported by the National Natural Science Foundation of China (Nos. 11601337, 11971311,11531001 and 11971319), the Foundation of Shanghai Normal University (No. SK201602) and the Foundation of Shanghai Municipal Education Commission, and the Montenegrin-Chinese Science and Technology Cooperation Project (No.312).
} 
and $v$ in graph $G$. Clearly, $D(G)$ is a symmetric matrix and its eigenvalues are real, denoted by $\lambda_{1}(G) \geq \lambda_{2}(G) \geq \cdots \geq \lambda_{n}(G)$. Moreover, $\lambda_{1}(G)$ is called the distance spectral radius of $G$ and is denoted by $\lambda(G)$. Further, Indulal, Gutman and Vijaykumar [15] in 2009 introduced distance energy which is defined as

$$
E_{D}(G)=\sum_{i=1}^{n}\left|\lambda_{i}(G)\right|
$$

They obtained some sharp bounds for the distance spectral radius and D-energy of graphs with diameter 2. Andelić, Koledin and Zoran Stanić in [2] obtained the exact value of the distance energy of several types of graphs. Recently, Varghesea, So and Vijayakumarc [23] studied how the distance energy of complete bipartite graphs changes when an edge is deleted. Vaidya and Popat [22] studied the distance energy of two particular graph compositions. Recently, Stevanovíc [20] gave more results on the graph composition.

A clique tree is a graph whose blocks are cliques (for example see [4]), a clique path $\mathbb{P}_{n_{1}, n_{2}, \cdots, n_{k}}$ is the graph obtained from the path $P_{k+1}=v_{1} \cdots v_{k+1}$ with order $k+1$ replacing $v_{i} v_{i+1}$ by $K_{n_{i}}$ such that

$$
V\left(K_{n_{i}}\right) \cap V\left(K_{n_{i+1}}\right)=v_{i+1}, i=1,2, \cdots, k-1 \text { and } V\left(K_{n_{i}}\right) \cap V\left(K_{n_{j}}\right)=\emptyset \text { if } i \neq j .
$$

Let $n_{+}(D(G)), n_{-}(D(G))$ denote the the number of positive and negative eigenvaues of $D(G)$, respectively. Lin, Liu and Lu [17] proved that

Theorem 1.1 [17] Let $G$ be a clique tree with order $n$. Then $n_{+}(D(G))=1$ and $n_{-}(D(G))=n-1$.

Furthermore, they proposed the following conjecture.

Conjecture 1.2 [17] Among all clique trees with cliques $K_{n_{1}}, \ldots, K_{n_{k}}$, the graph attains the maximum distance energy is $\mathbb{P}_{\left\lfloor\frac{n-k+3}{2}\right\rfloor, 2, \cdots, 2,\left\lceil\frac{n-k+3}{2}\right\rceil}$.

From theorem 1.1, the distance energy of a clique tree $G$ satisfies

$$
E_{D}(G)=\sum_{i=1}^{n}\left|\lambda_{i}(G)\right|=\lambda_{1}(G)-\sum_{i=2}^{n} \lambda_{i}(G)=2 \lambda_{1}(G),
$$

the last equation is from that the trace of $D(G)$ is $\sum_{i=1}^{n} \lambda_{i}(G)=0$. Thus it is sufficient to consider maximum spectral radius of clique trees instead of maximum distance energy of clique trees.

In addition, Zhang [27] studied the relation between the inertia and the distance energy for the line graph of unicyclic graphs. Moreover, Consonni and Todeschini [9] investigated the distance energy in terms of some invariants. Drury and Lin [10] characterized all connected graphs with distance energy in $[2 n-2,2 n]$. On the distance spectral radius of graphs, Liu [19] investigated the graphs with minimal distance spectral radius among the graphs with fixed vertex connectivity, matching number and chromatic number. Ilić 14 characterized the minimal spectral radius among trees given matching number. Bose, Nath and Paul [5] studied the connected graph with minimal distance spectral radius among all graphs fixed number of pendent vertices. Zhang [25] explored the graph with minimum distance spectral radius among all connected graphs with given diameter. Lin and Shu [18] gave some sharp lower and upper bounds for the distance spectral radius and characterized the graphs with the spectral radius attaining the bounds. Lin and Feng [16] characterized 
the connected graphs with minimal or maximal distance spectral radius among all graphs with fixed independence number.

The main purpose of this paper is to gave a positive answer of conjecture 1.2 .

\section{Proof of Conjecture 1.2}

Let $A$ be a symmetric matrix, $Y=\{1,2, \cdots, n\}$ be the index set of rows and columns of matrix $A$ and $\mathbb{1}_{n}$ be the all ones vector with $n$ elements, written by $\mathbb{1}$ if no ambiguity. Suppose $\left\{Y_{1}, Y_{2}, \cdots, Y_{m}\right\}$ is a partition of the set $Y$, the matrix $B=\left(b_{i j}\right)$ is called quotient matrix of $A$ corresponding to the partittion, where $A_{i, j}$ is a submatrix of $A$ corresponding to the row index and column index $Y_{i}$ and $Y_{j}$ respectively and $b_{i j}$ is the average row sum of matrix $A_{i, j}$. The $n \times m$ matrix $S=\left(s_{i j}\right)$ is called characteristic matrix, where $s_{i j}=1$ if $i \in Y_{j}$ and $s_{i j}=0$ if $i \notin Y_{j}$. The partition is called equitable partition if $A_{i, j} \mathbb{1}=b_{i j} \mathbb{1}$. For more details, the readers can refer to $[6]$.

Lemma 2.1 [6] Let $B$ be the quotient matrix of $A$ corresponding to an equitable partition. If $v$ is an eigenvector of $B$ for an eigenvalue $\mu$, then $S v$ is an eigenvector of $A$ for the same eigenvalues $\mu$, where $S$ is the characteristic matrix corresponding to the equitable partition.

\section{Corollary 2.2}

$$
\lambda\left(\mathbb{P}_{n_{1}+1,2, \cdots, 2, n_{2}+1}\right)=\lambda\left(B\left(\mathbb{P}_{n_{1}+1,2, \cdots, 2, n_{2}+1}\right)\right),
$$

where

$$
\begin{aligned}
& B\left(\mathbb{P}_{n_{1}+1,2, \cdots, 2, n_{2}+1}\right)=\left(\begin{array}{ccc}
n_{1}-1 & u_{k-1}^{T} & k n_{2} \\
n_{1} u_{k-1} & D\left(P_{k-1}\right) & n_{2} w_{k-1} \\
k n_{1} & w_{k-1}^{T} & n_{2}-1
\end{array}\right), \text { and } \\
& u_{k}=(1,2, \cdots, k)^{T}, \quad w_{k}=Q u_{k},
\end{aligned}
$$

$Q$ is a permutation matrix, the $(i, k+1-i)$-element of which is one, $1 \leq i \leq k$.

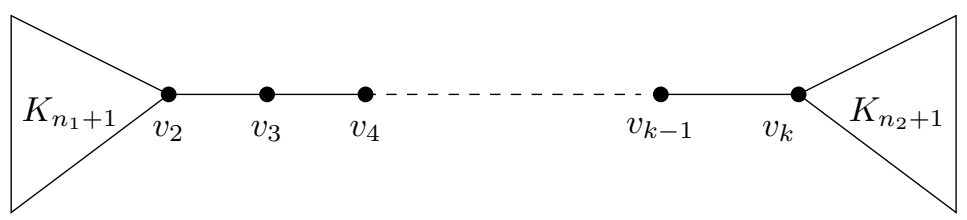

Fig. $1 \mathbb{P}_{n_{1}+1,2, \cdots, 2, n_{2}+1}$

Proof. Let $X_{1}, X_{2}, \cdots, X_{k}, X_{k+1}$ be a partition of $V\left(\mathbb{P}_{n_{1}+1,2, \cdots, 2, n_{2}+1}\right)$ with $X_{1}=V\left(K_{n_{1}+1}\right) \backslash\left\{v_{2}\right\}$, $X_{i}=\left\{v_{i}\right\}, i=2,3, \cdots, k$ and $X_{k+1}=V\left(K_{n_{2}+1}\right) \backslash\left\{v_{k}\right\}$. Then

$$
D\left(\mathbb{P}_{n_{1}+1,2, \cdots, 2, n_{2}+1}\right)=\left(\begin{array}{ccc}
(J-I)_{n_{1} \times n_{1}} & \mathbb{1} u_{k-1}^{T} & k J_{n_{1} \times n_{2}} \\
u_{k-1} \mathbb{1}^{T} & D\left(P_{k-1}\right) & w_{k-1} \mathbb{1}^{T} \\
k J_{n_{2} \times n_{1}} & \mathbb{1} w_{k-1}^{T} & (J-I)_{n_{2} \times n_{2}}
\end{array}\right) .
$$


The quotient matrix of $D\left(\mathbb{P}_{n_{1}+1,2, \cdots, 2, n_{2}+1}\right)$ corresponding to $X_{1}, X_{2}, \cdots, X_{k+1}$ is

$$
B\left(\mathbb{P}_{n_{1}+1,2, \cdots, 2, n_{2}+1}\right)=\left(\begin{array}{ccc}
n_{1}-1 & u_{k-1}^{T} & k n_{2} \\
n_{1} u_{k-1} & D\left(P_{k-1}\right) & n_{2} w_{k-1} \\
k n_{1} & w_{k-1}^{T} & n_{2}-1
\end{array}\right) .
$$

Moreover, $\left\{X_{1}, X_{2}, \cdots, X_{k+1}\right\}$ is an equitable partition. By lemma 2.1, $\lambda\left(\mathbb{P}_{n_{1}+1,2, \cdots, 2, n_{2}+1}\right)=$ $\lambda\left(B\left(\mathbb{P}_{n_{1}+1,2, \cdots, 2, n_{2}+1}\right)\right)$.

Now we consider the characteristic polynomial of the matrix $B\left(\mathbb{P}_{n_{1}+1,2, \cdots, 2, n_{2}+1}\right)$.

Lemma 2.3 If $x>\lambda\left(P_{k-1}\right)$ with $k>2$, then

(1) $u_{k-1}^{T}\left[x I-D\left(P_{k-1}\right)\right]^{-1} w_{k-1}=w_{k-1}^{T}\left[x I-D\left(P_{k-1}\right)\right]^{-1} u_{k-1}>0$;

(2) $u_{k-1}^{T}\left[x I-D\left(P_{k-1}\right)\right]^{-1} u_{k-1}=w_{k-1}^{T}\left[x I-D\left(P_{k-1}\right)\right]^{-1} w_{k-1}>0$;

(3) $\left\|u_{k-1}-w_{k-1}\right\|^{2}=\frac{(k-1) k(k-2)}{3}$;

(4) $u_{k-1}^{T}\left[x I-D\left(P_{k-1}\right)\right]^{-1}\left(u_{k-1}-w_{k-1}\right)=\frac{1}{2}\left(u_{k-1}-w_{k-1}\right)^{T}\left[x I-D\left(P_{k-1}\right)\right]^{-1}\left(u_{k-1}-w_{k-1}\right)$.

Proof. (1) Since $x>\lambda\left(D\left(P_{k-1}\right)\right)$ and $D\left(P_{k-1}\right)$ is a nonnegative symmetric matrix, we have

$$
\left[x I-D\left(P_{k-1}\right)\right]^{-1}=\frac{1}{x} \sum_{j=0}^{\infty}\left(\frac{D\left(P_{k-1}\right)}{x}\right)^{j},
$$

which is also a nonnegative symmetric matrix. Then

$$
u_{k-1}^{T}\left[x I-D\left(P_{k-1}\right)\right]^{-1} w_{k-1}=w_{k-1}^{T}\left[x I-D\left(P_{k-1}\right)\right]^{-1} u_{k-1}>0 .
$$

(2). By equation (2),

$$
u_{k-1}^{T}\left[x I-D\left(P_{k-1}\right)\right]^{-1} u_{k-1}>0 \text { and } w_{k-1}^{T}\left[x I-D\left(P_{k-1}\right)\right]^{-1} w_{k-1}>0 .
$$

Note that $w_{k-1}=Q u_{k-1}, Q^{T} Q=I$ and $Q^{T} D\left(P_{k-1}\right) Q=D\left(P_{k-1}\right)$, we have

$$
\begin{aligned}
w_{k-1}^{T}\left[x I-D\left(P_{k-1}\right)\right]^{-1} w_{k-1} & =u_{k-1}^{T} Q^{T}\left[x I-D\left(P_{k-1}\right)\right]^{-1} Q u_{k-1} \\
& =u_{k-1}^{T} \frac{Q^{T}}{x} \sum_{j=0}^{\infty}\left(\frac{D\left(P_{k-1}\right)}{x}\right)^{j} Q u_{k-1} \\
& =u_{k-1}^{T} \frac{1}{x} \sum_{j=0}^{\infty}\left(\frac{Q^{T} D\left(P_{k-1}\right) Q}{x}\right)^{j} u_{k-1} \\
& =u_{k-1}^{T} \frac{1}{x} \sum_{j=0}^{\infty}\left(\frac{D\left(P_{k-1}\right)}{x}\right)^{j} u_{k-1} \\
& =u_{k-1}^{T}\left[x I-D\left(P_{k-1}\right)\right]^{-1} u_{k-1} .
\end{aligned}
$$

(3). Divide into the following two cases to prove the assertion. 
Case 1: If $k$ is odd and $u_{k-1}-w_{k-1}=(2-k, \cdots,-3,-1,1,3, \cdots, k-2)^{T}$, then

$$
\begin{aligned}
\left\|u_{k-1}-w_{k-1}\right\| & =\sqrt{2\left(1^{2}+3^{2}+\cdots+(k-2)^{2}\right)} \\
& =\sqrt{2\left(\frac{(k-1) k(2 k-1)}{6}-2^{2}+4^{2}+\cdots+(k-1)^{2}\right)} \\
& =\sqrt{2\left(\frac{(k-1) k(2 k-1)}{6}-4\left(1^{2}+2^{2}+\cdots+\left(\frac{k-1}{2}\right)^{2}\right)\right)} \\
& =\sqrt{\left.2\left(\frac{(k-1) k(2 k-1)}{6}-\frac{(k-1) k(k+1)}{6}\right)\right)} \\
& =\sqrt{\frac{(k-1) k(k-2)}{3}} .
\end{aligned}
$$

Case 2: If $k$ is even and $u_{k-1}-w_{k-1}=(2-k, \cdots,-2,0,2,4, \cdots, k-2)^{T}$, then

$$
\begin{aligned}
\left\|u_{k-1}-w_{k-1}\right\| & =\sqrt{2\left(2^{2}+4^{2}+\cdots+(k-2)^{2}\right)} \\
& =\sqrt{2\left(4\left(1^{2}+2^{2}+\cdots+\left(\frac{k-2}{2}\right)^{2}\right)\right)} \\
& =\sqrt{\frac{(k-1) k(k-2)}{3}} .
\end{aligned}
$$

(4). Since

$$
\begin{aligned}
& \left(u_{k-1}-w_{k-1}\right)^{T}\left[x I-D\left(P_{k-1}\right)\right]^{-1}\left(u_{k-1}-w_{k-1}\right) \\
= & u_{k-1}^{T}\left[x I-D\left(P_{k-1}\right)\right]^{-1}\left(u_{k-1}-w_{k-1}\right)-w_{k-1}^{T}\left[x I-D\left(P_{k-1}\right)\right]^{-1}\left(u_{k-1}-w_{k-1}\right) \\
= & u_{k-1}^{T}\left[x I-D\left(P_{k-1}\right)\right]^{-1} u_{k-1}-u_{k-1}^{T}\left[x I-D\left(P_{k-1}\right)\right]^{-1} w_{k-1}-w_{k-1}^{T}\left[x I-D\left(P_{k-1}\right)\right]^{-1} u_{k-1} \\
& +w_{k-1}^{T}\left[x I-D\left(P_{k-1}\right)\right]^{-1} w_{k-1},
\end{aligned}
$$

then

$$
\left(u_{k-1}-w_{k-1}\right)^{T}\left[x I-D\left(P_{k-1}\right)\right]^{-1}\left(u_{k-1}-w_{k-1}\right)=2 u_{k-1}^{T}\left[x I-D\left(P_{k-1}\right)\right]^{-1}\left(u_{k-1}-w_{k-1}\right)
$$

which is from (1) and (2). So

$$
u_{k-1}^{T}\left[x I-D\left(P_{k-1}\right)\right]^{-1}\left(u_{k-1}-w_{k-1}\right)=\frac{1}{2}\left(u_{k-1}-w_{k-1}\right)^{T}\left[x I-D\left(P_{k-1}\right)\right]^{-1}\left(u_{k-1}-w_{k-1}\right) .
$$

Lemma 2.4 If $x>\lambda\left(P_{k-1}\right)$, then

$$
\begin{aligned}
& \operatorname{det}\left(x I-B\left(\mathbb{P}_{n_{1}+1,2, \cdots, 2, n_{2}+1}\right)\right) \\
= & {\left[(x+1)^{2}-\left(n_{1}+n_{2}\right)\left(1+\alpha_{k-1}\right)(x+1)+n_{1} n_{2}\left(1+\alpha_{k-1}+k+\beta_{k-1}\right)\left(1-k+\gamma_{k-1}\right)\right] } \\
& \cdot \operatorname{det}\left(x I-D\left(P_{k-1}\right)\right),
\end{aligned}
$$

where

$$
\begin{gathered}
\alpha_{k-1}=u_{k-1}^{T}\left(x I-D\left(P_{k-1}\right)\right)^{-1} u_{k-1}, \beta_{k-1}=u_{k-1}^{T}\left(x I-D\left(P_{k-1}\right)\right)^{-1} w_{k-1}, \\
\gamma_{k-1}=\frac{1}{2}\left(u_{k-1}-w_{k-1}\right)^{T}\left(x I-D\left(P_{k-1}\right)\right)^{-1}\left(u_{k-1}-w_{k-1}\right) .
\end{gathered}
$$


Proof. Since $x>\lambda\left(P_{k-1}\right)$, then $x I-D\left(P_{k-1}\right)$ is an invertible matrix. By lemma 2.3

$$
\begin{aligned}
& \operatorname{det}\left(x I-B\left(\mathbb{P}_{n_{1}+1,2, \cdots, 2, n_{2}+1}\right)\right) \\
= & \operatorname{det}\left(\begin{array}{ccc}
x-\left(n_{1}-1\right) & -u_{k-1}^{T} & -k n_{2} \\
-n_{1} u_{k-1} & x I-D\left(P_{k-1}\right) & -n_{2} w_{k-1} \\
-k n_{1} & -w_{k-1}^{T} & x-\left(n_{2}-1\right)
\end{array}\right) \\
= & \operatorname{det}\left(\begin{array}{ccc}
x-\left(n_{1}-1\right)-n_{1} \alpha_{k-1} & 0 & -k n_{2}-n_{2} \beta_{k-1} \\
-n_{1} u_{k-1} & x I-D\left(P_{k-1}\right) & -n_{2} w_{k-1} \\
-k n_{1}-n_{1} \beta_{k-1} & 0 & x-\left(n_{2}-1\right)-n_{2} \alpha_{k-1}
\end{array}\right) \\
= & \operatorname{det}\left(\begin{array}{ccc}
x-\left(n_{1}-1\right)-n_{1} \alpha_{k-1} & 0 & -k n_{2}-n_{2} \beta_{k-1} \\
0 & x I-D\left(P_{k-1}\right) & 0 \\
-k n_{1}-n_{1} \beta_{k-1} & 0 & x-\left(n_{2}-1\right)-n_{2} \alpha_{k-1}
\end{array}\right) \\
= & \cdot \operatorname{det}\left(x I-D\left(P_{k-1}\right)\right) . \\
= & {\left[(x+1)^{2}-\left(n_{1}+n_{2}\right)\left(1+\alpha_{k-1}\right)(x+1)+n_{1} n_{2}\left(1+\alpha_{k-1}+k+\beta_{k-1}\right) \cdot\right.} \\
& \left.\left(1-k+\alpha_{k-1}-\beta_{k-1}\right)\right] \cdot \operatorname{det}\left(x I-D\left(P_{k-1}\right)\right) .
\end{aligned}
$$

Moreover,

$$
\begin{aligned}
\alpha_{k-1}-\beta_{k-1} & =u_{k-1}^{T}\left(x I-D\left(P_{k-1}\right)\right)^{-1}\left(u_{k-1}-w_{k-1}\right) \\
& =\frac{1}{2}\left(u_{k-1}-w_{k-1}\right)^{T}\left(x I-D\left(P_{k-1}\right)\right)^{-1}\left(u_{k-1}-w_{k-1}\right)=\gamma_{k-1} .
\end{aligned}
$$

Then

$$
\begin{aligned}
& \operatorname{det}\left(x I-B\left(\mathbb{P}_{n_{1}+1,2, \cdots, 2, n_{2}+1}\right)\right) \\
= & {\left[(x+1)^{2}-\left(n_{1}+n_{2}\right)\left(1+\alpha_{k-1}\right)(x+1)+n_{1} n_{2}\left(1+\alpha_{k-1}+k+\beta_{k-1}\right)\left(1-k+\gamma_{k-1}\right)\right] } \\
& \cdot \operatorname{det}\left(x I-D\left(P_{k-1}\right)\right) .
\end{aligned}
$$

Lemma 2.5 Let $\mathbb{P}_{n_{1}+1,2, \cdots, 2, n_{2}+1}$ be a clique path with $k$ cliques, where $n_{1}$ and $n_{2}$ are nonnegative integers. Then

$$
\lambda\left(\mathbb{P}_{n_{1}+1,2, \cdots, 2, n_{2}+1}\right) \geq \lambda\left(P_{k}\right)>\frac{(k-1)(k+1)}{3} .
$$

Proof. Since $D\left(P_{k}\right)$ is a principal submatrix of $D\left(\mathbb{P}_{n_{1}+1,2, \cdots, 2, n_{2}+1}\right)$, we have $\lambda\left(\mathbb{P}_{n_{1}+1,2, \cdots, 2, n_{2}+1}\right) \geq$ $\lambda\left(P_{k}\right)>\frac{\mathbb{1}_{k}^{T} D\left(P_{k}\right) \mathbb{1}_{k}}{k}=\frac{(k-1)(k+1)}{3}$.

Lemma 2.6 17 Among all clique trees with cliques $K_{n_{1}}, \ldots, K_{n_{k}}$, the graph attains the maximum distance spectral radius is $P_{n_{1}^{\prime}+1,2, \ldots, 2, n_{k}^{\prime}+1}$

Now we are ready to prove conjecture 1.2 . 
Proof. By theorem 1.1 it is sufficient to prove that $\mathbb{P}_{\left\lfloor\frac{n-k+3}{2}\right\rfloor, 2, \cdots, 2,\left\lceil\frac{n-k+3}{2}\right\rceil}$ has the maximum spectral radius in the clique tree. By lemma 2.6, the graph which attains the maximum distance spectral radius is the clique path $\mathbb{P}_{n_{1}+1,2, \cdots, 2, n_{2}+1}$. If $n_{1}, n_{2}, n_{1}^{\prime}, n_{2}^{\prime}$ are positive integers such that

$$
\max \left\{n_{1}, n_{2}\right\}<\max \left\{n_{1}^{\prime}, n_{2}^{\prime}\right\} \text { and } n_{1}+n_{2}=n_{1}^{\prime}+n_{2}^{\prime}=n-1-k,
$$

which implies that $n_{1} n_{2}>n_{1}^{\prime} n_{2}^{\prime}$, we will show that

$$
\lambda\left(\mathbb{P}_{n_{1}+1,2, \cdots, 2, n_{2}+1}\right)>\lambda\left(\mathbb{P}_{n_{1}^{\prime}+1,2, \cdots, 2, n_{2}^{\prime}+1}\right) .
$$

Let $f\left(n_{1}, n_{2}, x\right)=\operatorname{det}\left(x I-B\left(\mathbb{P}_{n_{1}+1,2, \cdots, 2, n_{2}+1}\right)\right)$ be the characteristic polynomial of matrix $B\left(\mathbb{P}_{n_{1}+1,2, \cdots, 2, n_{2}+1}\right)$, where $B\left(\mathbb{P}_{n_{1}+1,2, \cdots, 2, n_{2}+1}\right)$ is a $(k+1) \times(k+1)$ matrix, similarly define $f\left(n_{1}^{\prime}, n_{2}^{\prime}, x\right)$. By the definition of $f\left(n_{1}, n_{2}, x\right), f\left(n_{1}^{\prime}, n_{2}^{\prime}, x\right)$ and lemma 2.4

$$
\begin{aligned}
f\left(n_{1}, n_{2}, x\right)-f\left(n_{1}^{\prime}, n_{2}^{\prime}, x\right)= & \left.\left(n_{1} n_{2}-n_{1}^{\prime} n_{2}^{\prime}\right)\left(1+\alpha_{k-1}+k+\beta_{k-1}\right)\left(1-k+\gamma_{k-1}\right)\right] \\
& \cdot \operatorname{det}\left(x I-D\left(P_{k-1}\right)\right) .
\end{aligned}
$$

Suppose $x \geq \lambda\left(P_{k}\right)>\lambda\left(P_{k-1}\right)$, by lemma 2.3 .

$$
\alpha_{k-1}, \beta_{k-1}>0 \text { and } \operatorname{det}\left(x I-D\left(P_{k-1}\right)\right)>0 .
$$

By the Perron-Frobenius theorem, there is an unique unit positive eigenvector $X=\left(x_{1}, x_{2}, \cdots, x_{k-1}\right)^{T}$ of $D\left(P_{k-1}\right)$ corresponding to $\lambda\left(P_{k-1}\right)$. Then

$$
\lambda\left(P_{k-1}\right)=X^{T} Q^{T} D\left(P_{k-1}\right) Q X=X^{T} D\left(P_{k-1}\right) X,
$$

which implies that $Q X$ is also an unit positive eigenvector of $D\left(P_{k-1}\right)$ corresponding to $\lambda\left(P_{k-1}\right)$, where $Q$ is a $(k-1) \times(k-1)$ permutation matrix whose $(i, k-i)$-element is one for $1 \leq i \leq k-1$. Thus $X=Q X$, that is, $x_{i}=x_{k-i}, 1 \leq i \leq k-1$. Hence

$$
X^{T}\left(u_{k-1}-w_{k-1}\right)=0 .
$$

Furthermore, by Courant-Fischer theorem (refer to theorem 4.2.6 in [13]),

$$
\begin{aligned}
\gamma_{k-1} & =\frac{1}{2}\left(u_{k-1}-w_{k-1}\right)^{T}\left[x I-D\left(P_{k-1}\right)\right]^{-1}\left(u_{k-1}-w_{k-1}\right) \\
& \leq \frac{1}{2}\left\|u_{k-1}-w_{k-1}\right\|^{2} \frac{1}{x-\lambda_{2}\left(D\left(P_{k-1}\right)\right)} .
\end{aligned}
$$

By $x \geq \lambda\left(P_{k}\right)>\lambda\left(P_{k-1}\right)$ and lemma 2.5 , we have

$$
x \geq \lambda\left(P_{k}\right)>\frac{(k-1)(k+1)}{3} .
$$

By theorem 1.1 and lemma 2.3 ,

$$
\lambda_{2}\left(D\left(P_{k-1}\right)\right)<0 \text { and }\left\|u_{k-1}-w_{k-1}\right\|^{2}=\frac{(k-1) k(k-2)}{3} .
$$

Inequalities (6), (7) and (8) implies that

$$
\gamma_{k-1} \leq \frac{(k-1) k(k-2)}{6} \frac{1}{x}<\frac{(k-1) k(k-2)}{6} \frac{1}{\frac{(k-1)(k+1)}{3}}<k-1 .
$$


Take inequalities (5), (9) and $n_{1} n_{2}>n_{1}^{\prime} n_{2}^{\prime}$ into (4),

$$
f\left(n_{1}, n_{2}, x\right)-f\left(n_{1}^{\prime}, n_{2}^{\prime}, x\right)>0 .
$$

Which implies that $f\left(n_{1}, n_{2}, x\right)-f\left(n_{1}^{\prime}, n_{2}^{\prime}, x\right)>0$ for $x \geq \lambda\left(P_{k}\right)$. Since $\lambda\left(\mathbb{P}_{n_{1}+1,2, \cdots, 2, n_{2}+1}\right) \geq \lambda\left(P_{k}\right)$ and $\lambda\left(\mathbb{P}_{n_{1}^{\prime}+1,2, \cdots, 2, n_{2}^{\prime}+1}\right) \geq \lambda\left(P_{k}\right)$, then

$$
\lambda\left(\mathbb{P}_{n_{1}+1,2, \cdots, 2, n_{2}+1}\right)>\lambda\left(\mathbb{P}_{n_{1}^{\prime}+1,2, \cdots, 2, n_{2}^{\prime}+1}\right) .
$$

Since $\left\lfloor\frac{n-k+1}{2}\right\rfloor,\left\lceil\frac{n-k+1}{2}\right\rceil$ are the only numbers satisfying that, for every positive integers $n_{1}^{\prime}, n_{2}^{\prime}$ and $n_{1}^{\prime}+n_{2}^{\prime}=n+1-k$ such that

$$
\begin{gathered}
\max \left\{\left\lfloor\frac{n-k+1}{2}\right\rfloor,\left\lceil\frac{n-k+1}{2}\right\rceil\right\} \leq \max \left\{n_{1}^{\prime}, n_{2}^{\prime}\right\} \text { and } \\
\left\lfloor\frac{n-k+1}{2}\right\rfloor+\left\lceil\frac{n-k+1}{2}\right\rceil=n_{1}^{\prime}+n_{2}^{\prime}=n+1-k .
\end{gathered}
$$

Thus $\mathbb{P}_{\left\lfloor\frac{n-k+1}{2}+1\right\rfloor, 2, \cdots, 2,\left\lceil\frac{n-k+1}{2}\right\rceil}+1=\mathbb{P}_{\left\lfloor\frac{n-k+3}{2}\right\rfloor, 2, \cdots, 2,\left\lceil\frac{n-k+3}{2}\right\rceil}$ has the maximum spectral radius in the clique tree.

\section{Acknowledgments}

The authors are grateful to the referees for their valuable comments and suggestions which lead to a great improvement of this paper.

\section{References}

[1] M. Aouchiche, P. Hansen, Distance spectra of graphs: a survey, Linear Algebra Appl., 458 (2014) 301-386.

[2] M. Andelić,T. Koledin, Z. Stanić, Distance spectrum and energy of graphs with small diameter, Appl. Anal. Discrete Math., 11 (2017) 108-122.

[3] R. B. Bapat,. Graphs and matrices, Second edition. Universitext. Springer, London, 2014.

[4] J. R. S. Blair, B. Peyton, An introduction to chordal graphs and clique trees. Graph theory and sparse matrix computation, 1-29, IMA Vol. Math. Appl., 56, Springer, New York, 1993.

[5] S. S. Bose, M. Nath and S. Paul, Distance spectral radius of graphs with $r$ pendent vertices, Linear Algebra and its Applications, 435 (2011) 2828-2836.

[6] A. E. Brouwer, W. H. Haemers, Spectra of graphs. Universitext. Springer, New York, 2012. xiv +250 pp.

[7] G. Caporossi, E. Chasset, B. Furtula, Some conjectures and properties on distance energy, Cah. GERAD, (2009), G-2009-64.

[8] Y.-J. Cheng, J. C.-H. Lin, Graph families with constant distance determinant. Electron. J. Combin., 25 (2018), no. 4, Paper 4.45, 21 pp. 
[9] V. Consonni, R. Todeschini, New spectral indices for molecule description, MATCH Commun. Math. Comput. Chem., 60 (2008) 3-14.

[10] S. Drury, H. Q. Lin, On graphs with near minimal distance energy. Linear Algebra Appl., 477 (2015) 76-101.

[11] R. L. Graham, L. Lovász, Distance matrix polynomials of trees. Adv. in Math., 29 (1978) 60-88.

[12] R. L. Graham, H. O. Pollak, On the addressing problem for loop switching, Bell System Tech. J., 50 (1971) 2495-2519.

[13] R. A. Horn and C. R. Johnson. Matrix analysis. Cambridge University Press, Cambridge, second edition, 2013.

[14] A. Ilić, Distace spectral radius of trees given matching number, Discrete Applied Mathematics, 158 (2010) 1799-1806.

[15] G. Indulal, I. Gutman, A. Vijaykumar, On the distance energy of a graph, MATCH Commun. Math. Comput. Chem., 60 (2007) 461-472.

[16] H. Q. Lin, L. H. Feng, The distance spectral radius of graphs with given independence number, Ars Combin., 121 (2015), 113-123.

[17] H. Q. Lin, R. F. Liu, X. W. Lu, The inertia and energy of the distance matrix of a connected graph, Linear Algebra Appl., 467 (2015), 29-39.

[18] H. Q. Lin, J. L. Shu, Sharp bounds on distance spectral radius of graphs, Linear Multilinear Algebra, 61 (2013) 442-447.

[19] Z. Z. Liu, On spectral radius of the distance matrix, Applicable Analysis and Discrete Mathematics, 4 (2010) 269-277.

[20] D. Stevanović, Comments on "Some New Results on Energy of Graphs", MATCH Commun. Math. Comput. Chem., 80 (2018) 445-447.

[21] D. Stevanović, A. Ilić, Spectral properties of distance matrix of graphs, in: I. Gutman, B. Furtula (Eds.), Distance in Molecular Graphs Theory, in: Math. Chem. Monogr., vol.12, University of Kragujevac, Kragujevac, 2010, pp.139-176.

[22] S. K. Vaidya, K. M. Popat, Some new results on energy of graphs, MATCH Commun. Math. Comput. Chem., 77 (2017) 589-594.

[23] A. Varghese, W. So, A. Vijayakumar, Distance energy change of complete bipartite graph due to edge deletion, Linear Algebra Appl., 553 (2018) 211-222.

[24] S.-J. Xu, R. Gysel, D. Gusfield, Minimum average distance clique trees, SIAM J. Discrete Math., 29 (2015) 1706-1734.

[25] X. L. Zhang, On the distance spectral radius of some graphs, Linear Algebra and its Applications, 437 (2012) 1930-1941. 
[26] X. L. Zhang, The inertia and energy of distance matrices of complete k-partite graphs, Linear Algebra Appl., 450 (2014) 108-120.

[27] X. L. Zhang, Inertia and distance energy of line graphs of unicyclic graphs, Discrete Appl. Math., 254 (2019) 222-233.

[28] B. Zhou, A. Ilić, On distance spectral radius and distance energy of graphs, MATCH Commun. Math. Comput. Chem., 64 (2010) 261-280. 